\title{
DISCURSOS PARA LA “SOCIEDAD DE LA INFORMACIÓN": UN ANÁLISIS CRÍTICO SOBRE CIUDADES DIGITALES EN ARGENTINA.
}

Ana Gabriela Yeremian ${ }^{1}$. Universidad Nacional de Córdoba. Argentina. gabriela.yeremian@gmail.com

Georgina Lía Remondino. Universidad Nacional de Córdoba. Argentina. georgina.remondino@gmail.com

\section{RESUMEN}

Este trabajo presenta una mirada crítica sobre aquellos discursos que desde diversos ámbitos promocionan a la llamada "Sociedad de la Información" (SI). Atendemos a ellos porque motivan en la actualidad prácticas particulares que modifican formas de intervención en el espacio público, como es el caso de las "ciudades digitales". En un primer momento reflexionamos -en base a aportes de la filosofía de la técnica, de la sociología y la comunicación social- sobre las promesas de la SI como modelo de sociedad y el valor que en él adquieren las "tecnologías de la información y la comunicación" (TICs). Sostenemos que la SI y las TICs se encarnan en dispositivos, prácticas y discursos situados en sociedades y actores concretos. Seguidamente, analizamos algunos proyectos de "ciudades digitales" en Argentina que resultan de especial interés para revisar los modos en que la SI permea políticas públicas, generando sentidos en relación a los modos de concebir a la ciudad, a los actores sociales y a su vínculo con las instancias gubernamentales. En las conclusiones compartimos algunas reflexiones sobre las modalidades con que estos discursos delimitan "lo público" y en la centralidad que adquiere la comunicación para pensar las ciudades y los modos de vida contemporáneos.

PALABRAS CLAVE: Ciudades digitales - Sociedad de la Información - Discursos TIC's.

\footnotetext{
${ }^{1}$ Autor correspondiente

Ana Gabriela Yeremian: Becaria doctoral, Centro de Investigaciones y Estudios sobre Cultura y Sociedad (CIECS-CONICET), Universidad Nacional de Córdoba, Argentina.

Correo: gabriela.yeremian@gmail.com
} 


\title{
DISCOURSES FOR "INFORMATION SOCIETY": A CRITICAL ANALYSIS OF DIGITAL CITIES IN ARGENTINA.
}

\begin{abstract}
This work presents a critical look at those discourses that from various areas promote the "Information Society" (IS). We focus on these discourses because they are currently motivating particular practices that modify intervention ways and observation of different actors in the public place, such as the "digital cities". To start with, we reflect - based on technical philosophy, sociology and social communication contributions - about IS promises as a society model and the value acquired by "information and communication technologies" (ICTs). We believe that the Information Society and the ICTs are reproduced in mechanisms, practices, and discourses situated in particular societies and actors. In our second step we reflect on some "digital city" models in Argentina that turn out to be of special interest to analyze critically the way in which the IS permeates public policies, generating effects of meaning related to ways of conceiving the city, the social actors and their links in the governmental instances. In the conclusion we share some reflections about the particular ways in which these discourses define the public sphere and the centrality that the communication gains to think about cities and contemporary ways of life.
\end{abstract}

KEY WORDS: Digital Cities - Information Society - Discourses - ICTs.

\section{INTRODUCCIÓN}

Este artículo presenta una mirada crítica sobre aquellos discursos que desde diversas políticas públicas, estrategias de mercado y actores sociales promocionan a la llamada "Sociedad de la Información" (SI). Atendemos a estos discursos porque ellos motivan en la actualidad prácticas particulares que modifican las formas de intervención y visibilización en el espacio público. En este sentido, podemos señalar el auge y desarrollo de diferentes proyectos de "ciudades digitales" entendidas como una modalidad específica de vinculación tecnológica al colocar en relación a diferentes actores sociales en función de cierto desarrollo científico-tecnológico; siendo el componente central en esas experiencias las llamadas "nuevas tecnologías de la información y la comunicación" (TICs), especialmente Internet. En la actualidad los organismos gubernamentales y de financiamiento nacional e internacional requieren alguna modalidad de vinculación tecnológica en las políticas públicas que promocionan. Encontramos entes -como la UNESCO a nivel internacional, empresas privadas como L'Oreal, Universidades Estatales y agencias de investigación científica a nivel nacional- que seleccionan y/o participan en la formulación de proyectos de investigación y desarrollo, transmisión de tecnología y asistencia técnica que implican algún tipo de vinculación tecnológica dentro de sus fundamentos u objetivos. Por su parte, los potenciales destinatarios/beneficiarios de sus planes de fomento económico 
y avales institucionales van desde pequeñas y medianas empresas hasta gobiernos locales e instituciones barriales. Sin duda este abanico de instituciones y de relaciones de fomento posible encuentra en común el valor positivo asignado a las tecnologías en diversas esferas de la sociedad.

Es por ello que atendemos con especial consideración a los proyectos de "ciudades digitales", pues estos proyectos locales encuadran dentro de las tendencias políticas y económicas a nivel global; y como casos de estudio permiten trazar algunas relaciones e interpretaciones con un horizonte de estudio más amplio.

Específicamente, aquí nos interesa poner de relieve la dimensión socio-comunicativa y cultural de esos procesos de informatización que se encaran con los proyectos de "ciudades digitales", aspectos muchas veces no contemplados en otros trabajos que se interesan por el fenómeno. Abordando esta dimensión, la de la comunicación y la cultura, pretendemos ofrecer otra mirada acerca de los discursos que atraviesan los procesos de construcción de políticas públicas y de desarrollo tecnológico que impulsan diferentes sectores sociales. Para ello, focalizamos en rastrear los sentidos y las significaciones sociales sobre las SI y las TICs que acompañan y legitiman las propuestas y el desarrollo de "ciudades digitales" desde el caso particular de dos proyectos diferentes que se encaran actualmente en Argentina.

La ciudad se vuelve objeto de interés para el campo de estudio de la comunicación en cuanto se aborda la "puesta en común" que excede la centralidad de los medios de comunicación; y a partir de entonces se posa la mirada también sobre la ciudad como co- constitutiva de las prácticas de comunicación, y como espacio material y simbólico de producción, circulación y apropiación de sentidos socialmente compartidos. La ciudad puede ser pensada entonces no desde su aspecto territorial sino como una red de interacciones que entrelaza y marca "modos de estar juntos" en un espacio común. La ciudad construye lazo social desde prácticas comunicativas marcadas por posiciones estructurales diferenciales (como la clase, edad, género, etc.) de los sujetos que la hacen existir pero que a la vez son condicionados por ésta ${ }^{2}$. Como señala Varela, "porque la comunicación tiene que ver con las prácticas del convivir, quizás el modo de estar juntos paradigmático de nuestra cultura contemporánea sea el de hacer

\footnotetext{
2 En El declive del hombre público (1974) Richard Sennett desarrolla las condiciones de vida de la burguesía y los procesos de cohabitación en el espacio urbano a partir de las reglas de cortesía que marcaban formas diferenciales de estar juntos y a la vez separados; distinguiendo los espacios públicos y los privados y sus prácticas asociadas. El autor inglés analiza cómo estos parámetros entran en crisis en la vida urbana de fin de siglo XX y privan al hombre de su espacio público. También Philippe Aries y Georges Duby en Historia de la vida privada (1989) muestra cómo la cultura burguesa fue demarcando formas de cohabitación y construcción del espacio urbano a partir de ciertas condiciones materiales, políticas y sociales que incidieron sobre esta clase. Por su parte, Jürgen Habermas en Historia y crítica de la opinión pública ([1962] 1994) desarrolla las condiciones de surgimiento y el desarrollo de una publicidad burguesa que redefine lo público y lo privado y en donde la ciudad comienza a cumplir nuevas funciones (principalmente como espacio de tráfico de mercancías y noticias) vinculadas a la constitución del Estado moderno y del nuevo modo de producción capitalista. El autor explica que, con el avance de este modelo de sociedad, el espacio público sufre una marcada transformación a partir de la aparición de los medios de comunicación masiva.
} 
ciudad y de ser hechos por ella" (Varela, 2003, p. 148).

En este marco, entendemos a la ciudad como el espacio en el cual se tejen formas diferentes y desiguales- de vivir y de dar sentido. Es en esta esfera entonces donde individuos y grupos, con posiciones y poderes diferenciales, construyen, disputan y negocian el orden simbólico de la vida en sociedad. Es ese orden, sus producciones simbólicas y esas formas de poder -que instalan las diferencias y rigen las negociaciones y disputas- lo que resulta de particular interés y pertinencia para una perspectiva comunicacional de la ciudad y de las políticas públicas en torno a ellas.

A ello se suma, por otra parte, el rol cada vez más preponderante que asumen las tecnologías comunicacionales en la reconfiguración de las ciudades y el lugar que ocupan como significantes que logran articular nuevos sentidos acerca de los modos de habitar e intervenir en el espacio urbano. Esta dominancia remite a las relaciones sociales, a aquellas pujas de sentido, en el marco de las cuales emergen el sentido y la función social asignada a estos dispositivos. Como señala Caletti, "en rigor, la tecnología es nada sino apenas una condensación de relaciones sociales. Muy probablemente pueda decirse, así, que refuerza la orientación y las reglas de esas relaciones sociales que han podido condensar en ella sus modos de vinculación con el mundo" (Caletti, 2002, p. 33).

\subsection{Premisas tecnológicas.}

Partimos de considerar que la Sociedad de la Información y las Tecnologías de la Información y la Comunicación se encarnan en dispositivos, prácticas y discursos situados en sociedades y actores concretos. En este sentido, en las sociedades modernas, las tecnologías -cualquiera sea el aparato técnico y el saber técnico que ponen en juego- impregnan todas las esferas de la vida individual y colectiva. Al respecto, entendemos que esta presencia indiscutida no puede ser pensada simplificadamente, tomando a las TIC como meros instrumentos sujetos a un fin determinado, o sin mayor poder que el de su aplicación inmediata ${ }^{3}$. Si bien es cierto que la creación de aparatos tecnológicos encuentra en los mercados económicos un estímulo importante que forma parte de sus condiciones de producción; también deben considerarse los valores culturales que dinamizan esos mercados. Así, por ejemplo, en este modelo de sociedad, la información se ha vuelto un bien de cambio valuable en términos monetarios, por ello las TICs hallan capitales de inversión para la investigación y producción científica en la materia. Ello es posible por la existencia de un valor cultural -el valor de la información- que sumerge a las tecnologías en el horizonte socio-histórico de una época y las sujeta a sus valores, poderes y lógicas de producción y consumo.

\footnotetext{
${ }^{3}$ Una de las ideas que mayor difusión y permeabilidad tiene tanto a nivel de la doxa social como en los discursos publicitarios, educativos y políticos es que el crecimiento de los entornos tecnológicos supone un avance social. Esta perspectiva coincide con una mirada evolucionista de la relación tecnologías/sociedad y, consideraría que la Sociedad de la Información sería un estadio avanzado e ineludible en el desarrollo autónomo del saber técnico y de la producción tecnológica.
} 
Entonces, desde la perspectiva asumida en este trabajo, las tecnologías y el saber técnico no tienen una existencia autónoma cuya naturaleza técnica explicaría su funcionamiento y su presencia en cada sociedad. Esta tesis niega tanto la posibilidad de una autonomía ontológica de las tecnologías como la de un determinismo tecnológico sobre la sociedad. Según Feenberg este determinismo se apoya en las siguientes premisas: (a) el progreso técnico sigue un camino unilineal y evolutivo conforme a metas y fines preconfigurados en el aparato mismo; (b) las sociedades y las instituciones sociales deber adaptarse al desarrollo tecnológico en tanto éste es inminente e irrefrenable (Feemberg, 1991). Estas premisas suponen que las tecnologías son auto-generadoras de sí mismas; y que -en base a un supuesto funcionalista compartido- las instituciones sociales deben incorporarlas conforme a los propósitos a los que sirven y a las necesidades que resuelven. Estos son algunos de los supuestos asumidos tanto por nuestra doxa social como por los discursos políticos y administrativos para justificar la incorporación de tecnologías como una fuerza exógena que incide positivamente en la sociedad. Esta concepción olvida tanto la "influencia recíproca" entre sociedad y tecnologías, como la posibilidad de las sociedades de marcar sus propios destinos, más allá de la "orientación" a fines específicos que engendra cada aparato tecnológico.

Por el contrario, la imposibilidad de una independencia de los escenarios sociohistóricos y culturales que las condicionan nos permite pensar a las tecnologías desde las significaciones y sentidos que encarnan a partir del momento en que pueden ser creadas, imaginadas, hasta su producción y apropiación social. Tomando la teoría de lo imaginario de Cornelius Castoriadis, Daniel Cabrera sostiene que el horizonte social de nuestra época está constituido por "un imaginario tecnocomunicacional en relación con el cual se postula la 'sociedad de la información'. El núcleo de este imaginario tecnocomunicacional son las nuevas tecnologías de la información y de la comunicación" (Cabrera, 2006, p. 161). Este imaginario se constituye sociohistóricamente a partir de lo que el autor analiza como el fracaso de las creencias centrales propias de la modernidad: las creencias en el progreso y en las técnicas modernas. A mediados del Siglo XX, el ideal de un progreso social ilimitado y las creencias optimistas en el poder de las tecnologías modernas, muestran una clara fractura -la llamada crisis de la modernidad-, pero no desaparecen. Por el contrario estas significaciones se desplazan hacia otras: son las actuales ideas en torno al "desarrollo", a las "tecnologías" y a la "comunicación". Éstas constituyen medularmente a este imaginario tecnocomunicacional en el que las TICs ocupan un lugar central en las representaciones sociales del mundo, en las creencias, esperanzas y deseos de la sociedad contemporánea.

Al considerarlas como significaciones imaginarias nodales de la sociedad actual, las TICs y la SI están asociadas a ciertas promesas sociales que favorecen su reproducción simbólica y material. En este sentido, son un núcleo imaginario que condensa sentidos con un alto componente utópico. Es decir, lo son en tanto se articulan las virtudes de los nuevos dispositivos con la idea de cambio social, de modo tal que se imponen como los únicos modos posibles de futuro, asociando el progreso técnico al progreso social. A esta creencia Mattelart la ha denominado "tecnoutopía" (Mattelart, 1997). 
Con ello señala el lugar central que ocupa la tecnología como solución deseable y necesaria a los problemas sociales. Ubicadas en este optimismo tecnológico, la potencia de las TICs se evidencia, al mismo tiempo, a través la eficiencia tecnológica que demuestran en la vivencia individual.

Desde esa mirada positiva, es decir como esperanza o utopía, las TICs están asociadas a la horizontalidad, transparencia, fluidez, flexibilidad, autonomía de los sujetos y a la hipercomunicación (Schmucler, 1997). Este pensamiento, que se reproduce en todas las esferas de lo social, promueve la idea de una nueva sociedad: la SI, necesariamente "más solidaria, más abierta y más democrática" (Mattelart, 2002). Así se presenta como una sociedad "mejor", más transparente, sin los obstáculos físicos y temporales de anteriores modelos de sociedad, que favorece el igualitarismo y la posibilidad de inclusión social a través del acceso y uso masificado de dispositivos tecnológicos.

En cuanto a las TICs, éstas se promueven como "novedad permanente". Los discursos publicitarios son aquellos que poseen la función de promocionar esta aparente cualidad inmanente de las tecnologías, y también motorizan el engranaje de su comercialización. Estos discursos refieren al cambio y a "lo nuevo" pues, al estar vinculados a la producción repetitiva de un dispositivo técnico, al mismo tiempo, auguran que el futuro es mejor por ser "más" que el hoy. La premisa es que lo novedoso acerca al futuro y, por lo tanto, es bueno. Un ejemplo nos muestra cómo dos de las mayores empresas tecnológicas del mundo encarnan estos presupuestos en sus ideas sobre las ciudades digitales. Para la empresa Motorola "una ciudad digital es más que conectividad e infraestructura; es una administración pública moderna que hace uso inteligente de la tecnología y de las aplicaciones para ofrecer a sus habitantes nuevos servicios, más seguridad y una nueva perspectiva de ciudadanía" ${ }^{4}$.

Para esta empresa, la aplicación tecnológica moderniza, ofrece un cambio de vida, abre nuevas posibilidades $\mathrm{y}$, sobre todo, realiza el deseo de bienestar y un modo aparentemente mejor de ser ciudadano. Por su parte, Microsoft la define como aquella ciudad "en la que, utilizando los recursos que brindan la infraestructura de telecomunicaciones y de informática existentes, fundamentalmente Internet, ofrece a sus habitantes un conjunto de servicios digitales a fin de mejorar el nivel de desarrollo humano, económico y cultural de esa comunidad, tanto a nivel individual como colectivo" 5 . Para esta multinacional, las TICs replican mejorando la calidad de vida total del colectivo y de los sujetos individuales; de modo que no existe la posibilidad de efectos parciales o dimensiones de lo social que no se encuentren afectados por los efectos positivos de las tecnologías. En síntesis, tal como lo afirma Cabrera, "en los discursos públicos (publicidades, notas de promoción, etc.), los anuncios no presentan adelantos tecnológicos sino anticipaciones de uso, cambios en la vida cotidiana, impacto social a la manera de narraciones e historias" (Cabrera, 2006, p. 174).

\footnotetext{
${ }^{4}$ Fuente: Ranking Motora de Ciudades Digitales.

${ }^{5}$ Fuente: “Ciudades Digitales" de Microsoft.
} 
Para finalizar este apartado, diremos que podemos analizar a las TICs desde tres dimensiones posibles que las constituyen como conglomerados tecnológicos. Para Cabrera las TICs son un conjunto heterogéneo de aparatos, instituciones y discursos, que se requieren mutuamente para existir (Cabrera, 2006, p. 18). En primer lugar, en tanto aparatos, refieren a la convergencia de varias ramas técnicas: la informática, la microelectrónica, las telecomunicaciones y medios de comunicación y la optoelectrónica. Por su parte, como instituciones se vuelven visibles en las nuevas formas de organización y producción económica, de gobierno, nuevos mercados y formas burocráticas en torno a la tecnociencia, etc. Por último, en cuanto a su dimensión discursiva, el hacer y el decir tecnológico permiten la comprensión, la aceptación y el uso de los aparatos tecnológicos (Cabrera, 2006, p. 15).

De estas tres dimensiones analíticas, en el próximo apartado nos ocuparemos de la dimensión discursiva de las mismas. Analizamos entonces algunos discursos que promocionan, fomentan y componen un conglomerado tecnológico específico: las ciudades digitales. Las ciudades digitales pueden ser vistas ellas mismas como TICs en tanto en ella coexisten instituciones, aparatos y discursos que se necesitan mutuamente para existir. No obstante, en este trabajo nos centraremos en analizar el modo en que las TICs son promocionadas en relación con modelos específicos de ciudades, actores, gobiernos y sociedades que habilitan.

\subsection{El enfoque sobre los discursos.}

Consideramos que los proyectos de "ciudades digitales", que tiene como principal actor social interviniente al Estado -municipales y/o provinciales- en tanto generador y articular de los restantes agentes, se constituyen en un espacio privilegiado de registro de la construcción discursiva que la sociedad realiza en torno a la ciudad. En estos discursos sobre la ciudad operan -bajo una multiplicidad de modalidades y matices- diversas creencias, representaciones y deseos sociales que sintetizan los significantes "TICs" y "SI". En tanto nombre-expresión, cada uno de estos dos significantes funciona "como nombre vacío aplicable, y en el que caben diferentes objetos y realidades" (Cabrera, 2006, p. 165). Por ello, cabe desnaturalizar el funcionamiento de estos significantes como sujetos gramaticales y analizar en cada corpus discursivo concreto las "cristalizaciones" de sentidos que cada una condensa.

A este respecto, nos planteamos analizar dos casos paradigmáticos de proyectos de ciudades digitales en Argentina entendiéndolos como cristalizaciones de los sentidos sociales dominantes acerca dichas nociones. Para ello, nos basamos en un enfoque sociosemiótico y combinamos herramientas metodológicas del análisis del discurso. La perspectiva sociosemiótica se basa en una doble hipótesis según la cual todo fenómeno social tiene una dimensión significante constitutiva $\mathrm{y}$, a la inversa, toda producción de sentido está insertada en lo social (Verón, 1997). Según Verón, este doble anclaje sólo puede ser abordado si consideramos la producción de sentido como discursiva, es decir, como manifiesta en cierto soporte material que denominamos "discursos sociales". De este modo, en nuestro estudio buscamos dar cuenta de la 
producción de los sentidos sociales sobre la SI y las TIC a través de discursos que refieren a ellas.

Para ello, relevamos los documentos oficiales publicados en las páginas Web de los gobiernos de dos lugares seleccionados como casos por el valor heurístico que adquieren a los fines de esta investigación. El corpus final está integrado por actas, programas, proyectos y comunicados oficiales del gobierno de la provincia de San Luis -y sitios relacionados a éste, como el de la Universidad de La Punta, el del proyecto Autopista de la Información y el sito del evento San Luis Digital 2010- y por los mismos materiales discursivos del gobierno municipal de la Ciudad de Rosario. En el caso de San Luis se consideraron las informaciones del gobierno provincial dado que, a diferencia del caso de Rosario en la provincia de Santa Fe, las políticas de digitalización de la ciudad de San Luis están esencialmente ibrincadas con el proyecto provincial "Autopista de la Información" y esta ciudad es presentada como modelo piloto de aquéllas. Desde la perspectiva sociosemiótica asumida, estos documentos pueden ser entendidos como discurso político en tanto intervienen instituciones políticas que marcan las condiciones de producción social de dichos discursos (Verón, 1987).

Tomamos como criterio para la selección del corpus aquellos discursos publicados en estas webs entre mayo y octubre de 2010, y en los que -al menos una vez- hubiera referencia explícita a la "Sociedad de la Información y/o del Conocimiento" y a las "Ciudades Digitales" y a las "TICs". En el corpus se realizó un análisis a nivel del enunciado, reconociendo valoraciones, categorías centrales y metáforas -entre otrosque permiten abordar la cuestión de las significaciones dominantes.

Se presentan a continuación aquellas categorías analíticas que, consideramos, poseen mayor poder interpretativo -en relación a otras categorías que emergieron-, ya sea por su recurrencia en el discurso y/o por los efectos de sentido posible que generan sobre políticas, prácticas y discursos.

\section{METODOLOGÍA}

El presente artículo parte de una necesidad epistemológica inicial sustentada, en primer lugar, sobre una exhaustiva analítica de fuentes para describir el status quo de partida del objeto de nuestro estudio, la sociedad de la información y las ciudades digitales. Posteriormente analizamos algunos proyectos de dichas ciudades digitales en el caso de Argentina, que resultan de especial interés para revisar los modos en que la sociedad de la información genera políticas públicas que se resumen en modos de concebir a la ciudad, a los actores sociales y a su vínculo con las instancias gubernamentales.

Dentro de este artículo existe, como en toda investigación, una estrecha relación entre los métodos de investigación teórica y los procesos del pensamiento. Para llegar a la formación de conceptos hay diferentes métodos del conocimiento teórico, entre ellos el método lógico-abstracto, que permitió aislar, separar y determinar las cualidades 
esenciales que caracterizan a los diferentes objetos fenómenos y procesos, en este caso cada una de las ciudades digitales. También se usó el método analítico-sintético, mediante el cual se ha realizado un análisis primero y una síntesis después, descomponiendo el fenómeno de las ciudades creativas, como elemento de la sociedad de la información, en los elementos que lo integran para conocer sus particularidades $\mathrm{y}$, mediante la síntesis, integrarlas en un concepto global.

\section{ANÁLISIS Y DISCUSIÓN}

\subsection{Modelos de sociedad e imperativos tecnológicos.}

En un primer acercamiento, consideraremos los modos en que las distintas significaciones sobre la "Sociedad de la Información" permean a los discursos circulantes en el corpus analizado. La categoría "Sociedad de la Información" es la que aparece con mayor recurrencia para nombrar al modelo de sociedad atravesado medularmente por las tecnologías informáticas. En el caso de la ciudad de Rosario, ésta es la única categoría que se usa para nominar a esta sociedad; mientras que en el caso de San Luis también aparecen -aunque con menor recurrencia y como sinónimosla referencia a la "Sociedad de la Información y del Conocimiento" (SIC) y a la "Sociedad del conocimiento" (SC).

Podríamos animar como hipótesis que si bien estas tres categorías ("SI", "SIC" y "SC") están nombrando tres modelos distintos de sociedades altamente informatizadas, en el caso de San Luis, el uso de las mismas como significantes equivalentes hace presuponer una falta de posicionamiento único sobre el modelo de sociedad deseado; pues estas categorías -en los debates académicos y políticos internacionales- refieren a ideales distintos. En estos espacios de discusión sobre diferentes dimensiones y problemáticas que atraviesan a la sociedad actual, se han sentado posiciones diversas sobre los modos de nombrarlas y las implicancias políticas de cada una. Mientras que la idea de SI pondría el acento sobre los procesos de informatización y sus roles causales sobre nuevas economías y formas organizativas que derivan de éstas; la noción de SC intentaría desarrollar una visión más integral y considerar los fenómenos de informatización como un proceso esencialmente humano ${ }^{6}$. Es por ello que utilizar estas tres categorías como sinónimos estaría evidenciando la falta de un posicionamiento claro o único -al menos en los modos de nombrar- sobre el modelo de sociedad al que adscribe el proyecto.

Otra hipótesis interpretativa sobre las formas en que el proyecto de San Luis nombra a la SI, podría animar la intención de utilizar la categoría "Sociedad del conocimiento" para generar un efecto de sentido que enfatice que el proyecto provincial intenta desmarcarse de una visión tecnocéntrica de la sociedad. Es decir, podría significar un

\footnotetext{
${ }^{6}$ Sobre los debates suscitados al interior de la esfera política y académica sobre los distintos usos y significaciones de las nociones de "Sociedad de la Información" y "Sociedad del Conocimiento", véase la interesante síntesis que realiza Sally Burch en "Sociedad de la Información/Sociedad del Conocimiento". Disponible en: http://vecam.org/article518.html (Consultado el 7 de octubre de 2010).
} 
intento por poner de relieve aspectos culturales relacionados con el bienestar social, antes que dar peso a las tecnologías desde miradas más instrumentales y economicistas. No obstante ello, esta hipótesis podría descartarse pues, como veremos más adelante, en este proyecto la dimensión de la cultura ha sido reducida a tareas de "concientización" para el uso de las TICs; es decir, se la ha dejado reducida a una esfera simbólica y práctica que sirva a la penetración de estas últimas en diversas dimensiones sociales.

Por su parte, en el caso de Rosario, la referencia a la SI aparece con menor recurrencia en comparación con el proyecto de San Luis (en la primera, el corpus presenta a esta noción sólo dos veces). En el caso de la ciudad santafesina, el proyecto se centra más en la idea de TICs, antes que en el modelo deseado de vinculación de la ciudad con la SI. Aquí la asociación entre significantes es la siguiente: "SI-TICs-inclusión social". Con esta cadena significante, no se observa una caracterización detallada de la SI como "modelo deseado de sociedad" aunque, al igual que en el caso de San Luis, se acepta el "imperativo tecnológico" de perseguir la incorporación de la sociedad a un mundo desarrollado, identificado siempre con la "Sociedad de la Información".

Tal como lo especificamos en el segundo apartado -refiriendo a las premisas tecnológicas-, en ese imperativo las TICs han sido históricamente consideradas como la herramienta, el instrumento, que garantizaría la incorporación de colectivos y sujetos al mundo desarrollado y, por ende, a lo que se considera que es "el progreso" de las sociedades. La supremacía de las TICs como significante central en todo el corpus discursivo analizado muestra la "naturalización" que estos proyectos gubernamentales hacen de las TICs como medio principal para alcanzar esos estadíos de la sociedad considerados superiores. Lo interesante a destacar es que, si en todos estos discursos la SI es presentada como inminente y necesariamente beneficiosa, el acceso a las TICs entonces se asume como el medio indiscutible para alcanzar algo esencialmente bueno. Bajo esta idea, se actualiza el imaginario desarrollista bajo el cual sólo hay un modelo deseado de sociedad al cual debe aspirar toda política pública; $y$ es el identificado con los países altamente industrializados e informatizados ${ }^{7}$. En ese sentido, los procesos históricos quedan relegados, las historias silenciadas, y los mecanismos y estructuras de dominación -entre países y entre sujetos- invisibilizados bajo un único modelo social al cual aspirar. Esta idea que encierra el mito del progreso tecnológico es asumida en estos programas políticos, y bajo este argumento justifican los esfuerzos por implementar los planes de "ciudades digitales" que impulsan.

Estos discursos gubernamentales estarían evidenciando la aceptación de, al menos, uno de los supuestos sobre los que se apoya el determinismo tecnológico; como desarrollamos anteriormente, éste sería la admisión de que las sociedades y las instituciones sociales deber adaptarse al desarrollo tecnológico en tanto éste es

\footnotetext{
${ }^{7}$ De allí que estos discursos presenten a las TICs como sustancialmente buenas, apoyando esta posición con evidencias empíricas que muestran los resultados económicos positivos en sociedades altamente informatizadas del llamado "primer mundo". Véase en ambos.
} 
perentorio e irrefrenable y necesariamente bueno. Los siguientes discursos muestran esta idea:

"En este mundo globalizado es indiscutible que, posibilitando la libre accesibilidad a la información y las comunicaciones, se abre una puerta a la cultural, a la educación, al desarrollo personal y profesional y al campo laboral [...] el acceso a la red de redes es considerado como la principal ventana al mundo [...] por tal motivo, varios gobiernos del país y el mundo trabaja en el tema intentando acotar la polémica brecha digital" 8 .

San Luis: una provincia en busca de la prosperidad digital: La Provincia de San Luis cuenta con la infraestructura necesaria para generar un cambio radical que la incluya en la Sociedad de la Información y el Conocimiento. Esta infraestructura es la Autopista de la Información, la encargada de asegurar la llegada de conectividad a todo pueblo de más de veinte habitantes ${ }^{9}$.

En síntesis, como significante central del imaginario tecnocomunicacional, las TICs son pensadas con un poder omnipresente $\mathrm{y}$, en tanto son el engranaje central del desarrollo social, tienen efectos inevitables e intrínsecamente positivos para el conjunto de la sociedad. En este sentido, resulta interesante pensar en el fatalismo que este pensamiento conlleva; la imposibilidad de considerar a la relación sociedad/tecnologías por fuera de una correspondencia funcional y al desarrollo como ineludibles destino. En este mismo sentido, Schmucler afirma que la ideología occidental del progreso se encarnaría en el siguiente razonamiento: "la tecnología, producto del progreso, sirve al desarrollo. En la medida que el progreso es una tendencia de los seres humanos y sirve para su bienestar, es deseable. El desarrollo, producto del progreso, es deseable y es bueno" (Schmucler, 1997, p. 192). Sin embargo, como lo señala el mismo autor "[...] el razonamiento esbozado encierra cierto fatalismo: el mundo es así y no podría ser de otra manera. La historia queda marginada, los procesos negados. La subjetividad relegada" (Schmucler, 1997, p. 192).

\subsection{Las TICs, categoría nodal en el campo de significaciones}

\subsubsection{Panacea social}

Retomado la asociación entre significantes "SI-TIC-inclusión social", podríamos afirmar que las TICs aparecen en ambos casos como garantías de solución a todos los problemas sociales y como promesas de progreso posible. Los ejemplos anteriores muestran esa significación. En ese sentido, consideradas como panaceas sociales, el Estado asume que debe garantizar el acceso de todos los ciudadanos a las TICs. Es por ello que los Estados buscan, a través de sus proyectos de digitalización, que las TICs sirvan a la inclusión social y al desempeño de toda la ciudadanía en la SI. En ese

\footnotetext{
8 Fuente: Ordenanza N8.052 Municipalidad de Rosario.

9 Fuente: Proyecto Autopista de la Información
} 
sentido, el progreso técnico deviene progreso social. Veamos algunos ejemplos en estos enunciados:

La inclusión de las Tecnologías de la Información y Comunicación (TIC) son el mejor indicador de la mejora de la calidad de vida y el crecimiento económico [...] La integración de las TIC en todos los aspectos de la economía y de la sociedad está creando una economía relacionada con el fenómeno digital responsable de generar prosperidad y crecimiento económico ${ }^{10}$.

Rosario Ciudad Digital es un proyecto que utiliza los recursos de las nuevas tecnologías para brindar a los ciudadanos un conjunto de servicios inteligentes que mejoran la calidad de vida y aportan al desarrollo social, económico y cultural de los individuos y la comunidad ${ }^{11}$.

Como lo muestran estos discursos, las TICs son recursos, herramientas para mejorar todos los aspectos de la vida social (educación, salud, seguridad, empleo, economía, vida cotidiana, etc.); pero a la vez se presentan como un fin en sí mismo en tanto garantizan ese bienestar social. Esto último también se sostendría sobre la misma creencia en el determinismo tecnológico que desarrollamos anteriormente. En resumen, en tanto son aceptadas como garantías de solución a todos los problemas sociales, las TICs adquieren un lugar nodal en torno al cual van a constelar otras significaciones que circulan por distintos campos discursivos. Si aceptamos que a través de las TIC se podrá "mejorar" la educación, la calidad de vida de los ciudadanos, la salud, los servicios públicos, las empresas, etc.; entonces las TICs se vuelven un significante nodal en tanto ocupa un lugar de articulador y organizador de distintos campo de significaciones.

Reducidas las TICs a su faz funcional, estos discursos justifican su utilización en tanto sirven a todos los actores sociales como herramientas para una multiplicidad de fines. En ese sentido, se presentan como la mejor propuesta para articular e integrar al Estado con los diversos agentes sociales, a éstos últimos entre sí, y también para sostener la cohesión del lazo social y el "correcto funcionamiento" del sistema político. Veamos algunos ejemplos:

Así como Internet es la red de información más completa de la actualidad, la Autopista es el modo más eficiente en que comunidad y Gobierno pueden relacionarse [...] Para el Estado, es una herramienta de organización y administración. Para los ciudadanos, un medio de acceso a los servicios electrónicos ${ }^{12}$.

\footnotetext{
${ }^{10}$ Fuente: Proyecto San Luis Digital 2010.

${ }^{11}$ Fuente: Proyecto Rosario 2010 Ciudad Digital.

1211 Fuente: Proyecto Autopista de la Información.
} 
Se podrá mejorar la calidad de vida de los ciudadanos, las empresas, la educación y la disponibilidad de información, mejorar la seguridad pública y los sistemas del cuidado de la salud, facilitar la operatoria municipal y mejorar la provisión de servicios municipales. ${ }^{13}$

Esta concepción instrumental de las TICs habilita a que los Estados consideren que es necesario garantizar la conectividad de todos los ciudadanos y que ello es sinónimo de "igualdad de oportunidades". De ese modo, "conectividad" e "integración social" se presentan como categorías semejantes o, al menos, asociadas en forma inherente. Es decir, se propone la siguiente equivalencia: conexión-integración, ciudadanos con acceso a Internet-ciudadanos integrados. Es por ello que, a la vez que proponen distintos modelos para el desarrollo de la infraestructura informática y de telecomunicaciones necesaria para extender los servicios de Internet -sobre todo Wi-fi, también prevén gestiones en otras áreas como la educación y la cultura. Pero resulta particularmente interesante que en estos ámbitos la educación, la capacitación y la cultura quedan subyugados a áreas básicas para lograr que los ciudadanos adquieran destrezas necesarias para el manejo de las TICs. Es decir, del abanico de relaciones entre TICs, educación y cultura se restringen los proyectos a una alfabetización digital en pos de la mentada conectividad, acceso y uso de las TICs, sobre todo de Internet. Es decir, la educación y la cultura son invocadas en tanto permiten una adecuada integración a la SI y a la vida en las ciudades digitales.

Como adelantamos anteriormente, y en relación con este valor asignado a las TICs, en este esquema de pensamiento la cultura queda relegada a una herramienta para lograr la penetración de estos dispositivos en todas las esferas de la vida social. Al respecto, distintos pensadores, que toman como referencia los aportes de la Teoría de la Dependencia, consideran a las actividades culturales y a los medios de comunicación como factores importante en la aceptación y desarrollo del sistema dominante $\mathrm{y}$, específicamente, del sistema capitalista. Lo anterior explica la importancia asignada por dichos investigadores al examen de las empresas culturales, en especial, de las características institucionales de las empresas transnacionales de comunicación y a los modos en que las políticas públicas se integran a estos agentes económicos. Para uno de estos intelectuales, Heriberto Muraro, "la función de las comunicaciones sería, de acuerdo a ese modelo, la de encubrir la desigual distribución de los beneficios económicos y socializar a los agentes económicos en la dirección requerida por el procesos monopólico trasnacional" (Muraro, 1987, p. 92) o, como lo expresa Héctor Schmucler:

La implantación tecnológica permite mantener el esquema de dominación sobre las sociedades receptoras, aun cuando formalmente se haya establecido la independencia política [...] las tecnologías, y entre ellas las tecnologías de la comunicación, deberían ser evaluadas en relación con los modelos de desarrollo a los que se encuentran orgánicamente vinculadas (Schmucler, 1997,

${ }^{13}$ Fuente: Proyecto Rosario 2010 Ciudad Digital. 
p. 63).

Por ello, teniendo en consideración estas posiciones, la cultura entonces es uno de las dimensiones que debemos analizar en estos proyectos políticos ya que esta esfera es también un campo atravesado por disputas entre distintos agentes políticos que estarían asignando a las TICs formas diferenciales de apropiación y de promoción. En ese sentido, como adelantamos más arriba, aquí advertimos que en ambos proyectos la dimensión de la cultura es referida desde una faz instrumental sujeta a fines operativos para difundir y facilitar el uso de las TICs. En ese sentido, la cultura es relegada a los fines operativos de cada proyecto. En el caso de Rosario, la esfera cultural es asociada al plano educativo y, en ese campo, el desafío principal es el generar propuestas educativas que garanticen el acceso y uso de las TICs a todos los ciudadanos. El supuesto de base es que la educación digital facilitará el éxito del proyecto propuesto. Por su parte, el proyecto de San Luis refiere a la necesidad de una "concientización" y "capacitación" de los ciudadanos comprometidos con la ejecución del proyecto. En ese sentido, la cultura y el campo de la educación son integrados vertical y jerárquicamente con un modelo de sociedad propuestos a los ciudadanos desde el gobierno y sobre el cual se espera que éstos logren "concientizar/se" aceptando las premisas y prácticas ofrecidas. En ese sentido, uno de los modelos de sociedad que aparecen en estos discursos aquel en el que el Estado estimula el acceso y uso de las TICs a través de la educación para facilitar la implantación y éxito de sus planes de gobierno. No aparecen aquí marcas sobre modelos educativos horizontales en los que sean los diversos sectores ciudadanos los que elaboren sus propuestas de apropiación de las TICs desde la educación y la cultura. Los siguientes fragmentos discursivos permiten identificar estas posiciones presentes en ambos proyectos:

La educación digital es una de las principales herramientas para hacer el proyecto democrático y equitativo, multiplicando las oportunidades de acceso, uso y apropiación de nuevas tecnologías, propiciamos mayores oportunidades sociales y económicas a más sectores de la comunidad y acortamos la brecha digital existente ${ }^{14}$.

Una cultura digitalizada: Los seis ejes de esta agenda [...] convergen en un mismo objetivo: generar los cambios necesarios que permitan a la sociedad sanluiseña incluirse en una próspera economía digital". "La Autopista es el fruto de un trabajo de años y de cientos de personas, que se han capacitado para ponerla en marcha, para mantenerla y para enseñar su aplicación. Al mismo tiempo, otros miles participaron de los diferentes cursos y talleres, cuyos contenidos tuvieron que ver fundamentalmente con el manejo de los programas informáticos ${ }^{15}$.

\footnotetext{
14 Fuente: Proyecto Rosario 2010 Ciudad Digital.

${ }^{15}$ Fuentes: Proyectos San Luis Digital 2010 y Autopista de la Información.
} 


\subsection{El carácter ideológico de la transparencia de las TICs}

Otro campo discursivo considerado en el corpus es el de las estructuras administrativas de gobierno. Bajo el mismo supuesto sobre las TICs -como facilitadoras de soluciones a los problemas que se pueden presentar en la ciudad-, también se las propone como dispositivos "transparentes" que permiten el acceso público a informaciones, permiten al Estado brindar seguridad y optimizar sus mecanismos de control $\mathrm{y}$, por ende, mejorar las relaciones -sin demasiadas mediaciones más que las técnicas- entre el Estado y la ciudadanía. Sin bien en el caso de San Luis se prevé en el proyecto el desarrollo de acciones específicas de gobierno digital, éstas aparecen íntimamente ligadas al poder de "comunicación" que garantizan las TICs; no es el caso de Rosario, en el que se complejiza más este fenómeno. En el sitio oficial de Autopista de la Información de San Luis vemos cómo las TICs son consideradas en las acciones de gobernabilidad y ejercicio de la ciudadanía por su poder instrumental de asegurar la "conectividad pública y sin costos":

es posible brindar servicio de Internet, telefonía y servicios de valor agregado como gobierno electrónico, mail, etc. (la autopista de la información) se trata de un sistema que mantiene interconectados todos los edificios de gobierno, en todas las localidades, como si se tratara de teléfonos internos, es decir, sin costo alguno. Esto ahorra el abono del servicio de llamadas a terceros y posibilita la comunicación del usuario final libre de cargo y sin restricción de tiempo, con los distintos establecimientos estatales: educativos, policiales $\mathrm{y}$ gubernamentales ${ }^{16}$.

Por su parte, en el caso de Rosario, el proyecto enuncia dos dimensiones en las que se interviene: el "e-gobierno" y la "e-democracia", dimensiones que aparecen disociadas discursivamente. La primera dimensión prevé como acciones de "e-gobierno" a distintas tareas de "gestión" o "administración pública". Mientras que la segunda, desarrolla acciones informáticas de participación ciudadana y que tenderían a facilitar la "e- democracia". Sin embargo, en esta concepción del gobierno y del ejercicio de la ciudadanía, la "democracia electrónica" lograda a través de las TIC es reducida al "voto electrónico" para "garantizar mayores niveles de transparencia, fluidez y orden en el proceso eleccionario". No obstante, pese a esta reducción de las prácticas ciudadanas posibles, la creencia en la "transparencia" de las tecnologías y en su poder de "universalización" motivan enunciados como el siguiente: "[...] [Las TICs] proporcionan a los ciudadanos un beneficio en forma directa: acceso a estructuras de gestión de la ciudad, realización de trámites en línea, acceso a informaciones, políticas locales, regionales y nacionales, y participación en decisiones que conciernan el hábitat urbano" 17 .

\footnotetext{
${ }^{16}$ Fuente: Proyecto Autopista de la Información.

17 Fuente: Proyecto Rosario 2010 Ciudad Digital.
} 
Esta "transparencia" opera en un doble sentido: por una parte, y como señalábamos en la Introducción, la transparencia es experimentada en la eficacia tecnológica que demuestran estas herramientas en la vivencia individual. Característica que es puesta en crisis cuando se señala cotidianamente que "se cayó el sistema" o "colapsó el sistema". Asimismo, y más fundamentalmente, la transparencia alude a cierta ausencia de medicaciones ("un beneficio en forma directa"), sobre todo las medicaciones de la cultura. Frente a la densa y compleja trama sociocultural, las TIC se erigen sobre el supuesto de una comunicación aséptica y basada en un acuerdo social de sujetos iguales. Por el contrario, evidencia su carácter ideológico justamente allí donde intenta autovalidarse. En este sentido, Schmucler explica que:

La tecnología, en realidad, intenta marginarse del campo del discurso -lugar de la ideología y de la disputa- para erigirse como transparencia. Impone hablar de ella sólo desde ella misma, en un tautológico ser lo que es que la instala en un ámbito de sacralidad. Indiscutible. [...] En el límite, la tecnología es el medio en el que (no con el que) los seres humanos se constituyen como tales.(Schmucler, 1997, p. 43).

Para seguir avanzando en el análisis de esta relación entre las gestiones de gobiernos y la ciudadanía a partir de las TICs, en el siguiente apartado nos detendremos a considerar los distintos actores que estos discursos consideran parte de los proyectos de "ciudades digitales" y los roles y características que se les asignan a cada uno de ellos.

\subsection{Los actores de las ciudades digitales}

En cuanto al Estado, como dijimos anteriormente, dado el calificativo de panacea social que los discursos analizados asignan a las TICs, el rol del Estado pasa a ser el de promotor, administrador y agente que debe garantizar el acceso universal y sin costos a estas tecnologías para, de ese modo, abonar esta nueva forma de integración social. Debilitadas otras formas modernas de inclusión social -como el pleno empleo, la educación y el Estado de Bienestar- el Estado se propone como garante de este nuevo modo de integración a toda la ciudadanía. Es por ello que los programas analizados se estructuran considerando distintas esferas de la vida social, de modo tal de abarcar la totalidad de instituciones y ámbitos de la vida cotidiana que involucran a distintos sectores de la sociedad. En ese sentido, en ambos discursos, el Estado -ya sea en su forma de municipio o como estado provincial- aparece como sujeto de la enunciación explícito. Aunque, en el caso del municipio de Rosario, esa presencia está menos marcada que en el caso del omnipresente discurso del gobierno de San Luis y hay un borramiento mayor de las marcas del sujeto de la enunciación sobre el enunciado. Por el contrario, en el caso de San Luis el Estado provincial aparece con mayor recurrencia dentro de los enunciados pero casi no hay referencias a los municipios sino las localidades y ciudades en los que la gobernación provincial desarrolla sus acciones para la Autopista de la información. 
Esto último estaría marcando también un modelo distinto de gestión de las ciudades digitales en uno y otro caso. En el caso de Rosario esto podría evidenciarse en que los discursos refieren a todos los sectores de la sociedad civil con mayor recurrencia y explicitando con mayor detalle a qué sectores se convocan al proyecto. Mientras que en el caso de San Luis, hay una universalización de este sector bajo dos categorías: los ciudadanos (y utilizados como sinónimos la ciudadanía, los habitantes y la población).

Por el contrario, en el discurso rosarino se enuncian una "multiplicidad de actores: Estado, Sociedad Civil, organizaciones sociales, empresas privadas, académicos, todos bajo una misma visión y en pos de una ciudad mejor" 18 . Nótese, finalmente, cómo en este último discurso los distintos actores aparecen sin una escala de jerarquía clara, se los coloca a todos en un mismo espacio de colaboración y participación. Especulativamente, estas diferencias en los discursos podrían estar dando cuenta de dos modelos distintos para pensar el rol del Estado dentro de los proyectos de "ciudades digitales".

En cuanto al sector empresarial, existe una coincidencia en ambos discursos. Las empresas privadas aparecen como "agentes contratados" por el Estado para brindar servicios técnicos -generalmente mediante alianzas por licitación pública-, ya sea como operadores, proveedores informáticos y de telecomunicaciones, etc. Esto resulta particularmente evidente en la página del evento organizado por el gobierno provincial de San Luis, San Luis Digital 2010. La feria de empresas informáticas y de telecomunicaciones, internacionales y algunas locales, hace evidente las alianzas de la gobernación con ese sector empresarial. La otra modalidad de aparición del sector de empresas privada dentro del corpus discursivo es como "destinatarias" de la política pública. Por ejemplo, uno de los ejes del proyecto de "ciudad digital" de Rosario es el "Incubador de empresas y tecnologías" y se allí estos actores son considerados aliados estratégicos o socios corporativos en los aspectos siempre estrictamente técnicos.

Por último, en cuanto a otros sectores que componen la sociedad civil, en el caso de San Luis hay una referencia a actores académicos que, desde las estructuras del Estado provincial, gestionan y administran el proyecto Autopista de la Información. Esta marca que destaca el desempeño de académicos y profesionales dentro de las estructuras del Estado vuelve a avalar la anterior tesis de una presencia mucho más centralista de la gobernación provincial en comparación con el caso de Rosario.

En cuanto a las organizaciones civiles, en los dos proyectos aparecen enunciadas en forma general sin destacarse algunos particularismos o sectores específicos de injerencia. En todo caso, el significante "ciudadanos" es el que posee mayor recurrencia en los enunciados.

Nótese cómo éste es utilizado como una categoría que "unifica e iguala" dentro del siguiente enunciado:

${ }^{18}$ Fuente: Proyecto Rosario 2010 Ciudad Digital. 
¿Quiénes pueden hacer uso de la Autopista de la Información? Cualquier ciudadano de la provincia de San Luis. Debido a que cualquier ente Gubernamental dispone los beneficios otorgados por la Autopista, y existen Cibers AUI, disponibles para cualquier ciudadano distribuidos por toda la Provincia ${ }^{19}$.

Por su parte, en el caso del proyecto rosarino aparecen una mayor cantidad de significantes para referir a este sector: ciudadanía, población, comunidad, vecinos, etc. En esta cita se observa cómo operan como sinónimos:

Este proyecto aspira lograr una mayor democratización del acceso a la información a través de la promoción de las tecnologías de información y comunicación a todos los sectores de población. En este sentido, se está trabajando en la Planificación de programas de capacitación para la ciudadanía y en la facilitación del acceso de los vecinos y vecinas a puestos de Internet ${ }^{20}$.

Pese al uso de estas categorías como sinónimos, el significante principal -no sólo por sus reiteraciones sino por la contaminación semántica producida por la aparición de otros significantes como "democracia", "gobierno", "participación ciudadana"-, es el de "ciudadanía".

Finalmente, sobre este sector, ambos proyectos los consideran tanto destinatarios como actores partícipes en distintas iniciativas para el logro de las "ciudades digitales". No obstante ello, esa participación oscila entre el rol de "promotores" de las iniciativas del Estado y el rol de "consumidores" y "beneficiarios" de los servicios que incluye cada proyecto. Los siguientes enunciados muestran este rol asignado a la ciudadanía.

[...] es el modo más eficiente en que comunidad y Gobierno pueden relacionarse [...] Para los ciudadanos, [es] un medio de acceso a los servicios electrónicos". "Cualquier ente Gubernamental dispone los beneficios otorgados por la Autopista, y existen Cibers AUI, disponibles para cualquier ciudadano distribuidos por toda la Provincia ${ }^{21}$. Favorecer un nuevo marco de relaciones entre los ciudadanos y los servicios. ${ }^{22}$

De esta forma, en estos discursos se marca una nueva forma de relación de los ciudadanos con las gobernaciones: desde la "ejecución" de proyectos públicos diseñados por la administración del Estado y desde el consumo de bienes y servicios incluidos en las políticas públicas. Quizás en este punto se manifiesta con mayor

\footnotetext{
${ }^{19}$ Fuente: Proyecto Autopista de la Información.

${ }^{20}$ Fuente: Proyecto Rosario 2010 Ciudad Digital.

${ }^{21}$ Fuente: Proyecto Autopistas de la Información.

${ }^{22}$ Fuente: Proyecto Rosario 2010 Ciudad Digital.
} 
tensión la redefinición que se produce a partir de la operatoria de las TIC entre las nociones de "lo público" y lo "privado". Y ello está íntimamente relacionado con las denominaciones de "e-gobierno" y "e-democracia" que señalamos anteriormente. En los proyectos de "ciudades digitales" que venimos analizando, la esfera pública está fuertemente atada -aunque de modo implícito- a la noción de "lo público" como lo estatal. Estos aspectos serán recuperados en las conclusiones.

\section{CONCLUSIONES}

En este trabajo hemos realizado un recorrido analítico por las principales significaciones que se encarnan en los discursos de dos proyectos de "ciudades digitales" en Argentina. Estos proyectos funcionaron como casos de valor heurístico para desentrañar aquellas significaciones dominantes a nivel de los discursos políticos que atraviesan a las políticas públicas y justifican diversas iniciativas en pos de un mentado desarrollo tecnológico y social. Para ello, focalizamos en rastrear los sentidos y las significaciones sociales sobre las SI y las TICs que disponen y legitiman a las dos propuestas analizadas. En esta conclusión nos interesa compartir algunas reflexiones a partir de los análisis realizados en este recorrido.

Podríamos afirmar que desde una perspectiva comunicacional las ciudades contemporáneas están constituidas por un núcleo central que conforman los medios de comunicación, la comunicación masiva y las tecnologías informáticas. Este núcleo tecnológico es el que hoy es propuesto por estos programas políticos bajo las fuertes figuras de los polos, las redes y las autopistas informáticas que atraviesan, configuran y marcan a las regiones y ciudades contemporáneas.

En referencia a lo anterior, H. Schmucler y P. Terrero señalan que desde sus orígenes la ciudad es un espacio de comunicación, y esta característica ha permanecido inalterada:

“Lo que ha ido cambiando -a veces de manera drástica- es el alcance de lo que se entiende por comunicación. La concepción del espacio, la percepción del sentido de la ciudad, los modelos imaginados para la construcción del hábitat, los rasgos atribuidos a la cultura urbana, podrían ser vistos como variables derivadas de este principio estructurante de la ciudad: la comunicación" (Schmucler y Terrero, 1991, p. 2).

En una sociedad en la que la comunicación y las tecnologías que la posibilitan son el núcleo del imaginario sociohistórico que le es propio, los "modos de estar juntos", de ser "ciudadanos", de "vivir la ciudad" se encuentran medularmente marcados por las significaciones que encarnen a la comunicación. En este sentido, tal como lo exponen los dos autores anteriores, lo que hemos de preguntarnos inicialmente, entonces, es qué es la comunicación y qué es la comunicación en las ciudades en la actualidad. En los proyectos analizados, la comunicación y su faz tecnológica son entendidas como el tejido instrumental que une lo disperso, iguala en la diferencia, integra los fragmentos a una totalidad. En estos modelos de ciudad, la comunicación ininterrumpida entre 
instituciones, entre ciudadanos y de éstos con el Estado, es elevada a nivel de trama y tejido que la estructura. Sin embargo, las desigualdades y las diferencias sólo son pensabas al interior de estos espacios de comunicación como condiciones u obstáculos a superar por medio de la vinculación tecnológica. La diferencia parece borrarse, la ciudad se aplana asemejándose a un plano unidimensional de la misma; plano cuyas coordenadas son dibujadas por los flujos de información y los polos tecnológicos que en ella se elevan.

En otro sentido, hoy los programas políticos analizados proponen que los vínculos comunicacionales en las ciudades digitales son modos de vivir y de relacionarse de los ciudadanos entre sí pero, sobre todo, de éstos con distintas áreas administrativas del Estado. En ese sentido, esta relación del Estado con los ciudadanos y de éstos entre sí es posible en tanto éstos últimos pueden ser consumidores de medios de comunicación y, sobre todo de tecnologías informáticas. De allí que el lazo social en estos proyectos es representado como vínculos centralmente comunicacionales. Bajo este supuesto que reduce e invisibiliza otras dimensiones de las relaciones sociales (como las económicas, las políticas, las culturales), se prometen garantías de integración y de correcto funcionamiento del Estado en su faz administrativa. Desde esta posición, los ciudadanos son consumidores de distintos servicios informáticos, así los puntos neurálgicos de las ciudades son pantallas, cámaras y espacios -domésticos, privados y públicos- de acceso a las TICs. Las plazas, oficinas públicas y otros centros nodales ahora encuentran en Internet algunas representaciones informáticas que "virtualizan" algunas de sus actividades y, por lo tanto, transforman sentidos y prácticas que hasta la fecha sólo se gestaban en espacios públicos y co-presenciales.

En este sentido, la ciudad como lugar en el que se desarrolla la vida política/democrática deviene en espacio de "lo público" 23 que, para el caso de las "ciudades digitales", es redefinido a partir de la mediación tecnológica. En los discursos analizados "lo público", por un lado, hace referencia directa a "lo estatal": los servicios que las gobernaciones ofrecen, los proyectos de "e-gobierno" y de "edemocracia", el objetivo de "garantizar acceso", entre muchos otros significantes. Por otro lado, el sentido de lo público opera bajo la forma de "lo abierto, accesible y disponible". Por ello, Rabotnikof (2007) señala que este sentido es el que mejor vincula la dicotomía público-privado con el par inclusión-exclusión. Así lo evidencian las propuestas de "acortar la brecha digital", "garantizar acceso", ofrecer servicio gratuito de internet en los puntos nodales de las ciudades, entre otros. Conjuntamente, estas dos acepciones son articuladas gracias a las TIC, en tanto a través de ellas es posible entablar otro vínculo político y otras prácticas en el marco de las relaciones ciudadanía-Estado (primer sentido) y esta novedad es posibilitada por las TIC como herramientas disponibles y abiertas a todos (segundo sentido). Es en este sentido que entendemos, tal como señalamos en la Introducción, que los discursos de las "ciudades digitales" motivan ciertas prácticas que modifican las formas de intervención y visibilización en el espacio público.

\footnotetext{
${ }^{23} \mathrm{Al}$ referir a "lo público" recuperamos las acepciones que desarrolla Nora Rabotnikof en El espacio público y la democracia moderna (1997).
} 
Sin embargo, también hemos señalado, que este modo de concebir la ciudad como espacio público/político niega los antagonismos sociales y las relaciones de poder a través de las cuales se construye el orden simbólico de cada sociedad. En diversos momentos analíticos señalamos que a través de estos proyectos de digitalización de las ciudades, muchos de estos aspectos sufren un corrimiento e incluso un borramiento. Esto puede señalarse como uno de los principales efectos de sentido que las TIC generan y que están vinculadas a las promesas sociales que encarnan: transparencia, ausencia de conflictos, horizontalidad, fluidez, autonomía de los sujetos, eficacia práctica, entre otros. Sin embargo, con ello se desconocen otras dimensiones insoslayables de la vida colectiva, sobre todo, justamente las disputas y conflictos a partir de los cuales estos sentidos logran instalarse y ser aceptados socialmente.

Esta clausura simbólica habilita la aparición de un orden a partir del cual se construyen la SI y las TIC como dispositivos discursivos que tienden al sostenimiento y la consolidación del orden social existente. De este modo, el funcionamiento social de este imaginario tecnocomunicacional instituido impide visibilizar otras alternativas, favoreciendo su reproducción social y ocultando su condición sociohistórica. En este sentido, podemos mencionar algunas cuestiones relevantes. En primer lugar, a través de las "ciudades digitales" se naturaliza un único modelo de sociedad que, bajo diversas modalidades, se basa recurrentemente en el mismo presupuesto: una idea de progreso necesariamente positivo y ventajoso -al mismo tiempo inevitable- en el cual las TIC vienen a constituirse en su motor de desarrollo. De este modo, se uniformiza, se produce un efecto de homogeneidad social -a escala global y local- que desconoce los particulares procesos socio-históricos y políticos locales y la trama cultural en la cual se han forjado. Para alcanzar este modelo de sociedad, el Estado asume la responsabilidad de garantizar la igualdad de oportunidades y la inclusión social de los ciudadanos. De allí, la cadena equivalencial "SI-TIC-Inclusión social". Es decir ésta última es entendida como "inclusión digital".

En segundo lugar, pero íntimamente vinculado a lo anterior, estos proyectos de digitalización operan bajo un borramiento de las reales desigualdades sociales y los mecanismos y estructuras de dominación quedan invisibilizados bajo un único modelo social pretendidamente igualitario que convierte al ciudadano en consumidor. Es decir, bajo estos discursos se enuncia una apertura, acceso y disponibilidad social de ciertos espacios y recursos, pero que no articula en el nivel de las prácticas con un ejercicio ciudadano efectivo. Por el contrario, apela implícitamente a la idea de un posible consumidor - noción, por otro lado, más igualadora y abarcadora que la de ciudadanía- de aquello sobre lo que se enuncia marcando únicamente sus beneficios y potencialidades (como productos mercantilizados).

Por último, la centralidad significante de las TIC y la SI muestra otra evidencia en tanto ellas logran absorber y articular todo los componentes del campo discursivo analizado: así, la cultura es una "cultura digitalizada", la educación se vuelve "educación digital" y la sociedad del futuro es "una (próspera) economía digital", 
organizada bajo el régimen político de la "e-democracia". Así, los proyectos de "ciudades digitales" de los que nos hemos ocupado en este artículo se configuran no ya como discursos de sino como discursos para una Sociedad de la Información, es decir, para su consolidación y reproducción a escala local.

\section{BIBLIOGRAFÍA}

Aries, P. \& Duby G. (1989). Historia de la vida privada. Madrid: Taurus.

Burch, S. (2005). Sociedad de la Información/Sociedad del Conocimiento. Disponible en: http:/ / vecam.org/article518.html (Consultado el 7 de octubre de 2010).

Cabrera, D. (2006). Las nuevas tecnologías como creencias y esperanzas colectivas. Buenos Aires: Editorial Biblios.

Caletti, S. (2002). Comunicación, política y espacio público. Notas para repensar la democracia en la sociedad contemporánea. Buenos Aires: Borradores de trabajo.

Feemberg, A. (1991). Critical Theory of Technology. New York: Oxford University Press.

Habermas, J. ([1962] 1994). Historia y crítica de la opinión pública. México DF: Editorial Gustavo Gil.

Mattelart, A. (1997). Utopía y realidades del vínculo global. Para una crítica del tecnoglobalismo. En Revista Diálogos de la Comunicación, núm. 50 de octubre de 1997, pp. 9-25.

Mattelart, A. (2002). Historia de la sociedad de la información. Buenos Aires: Paidós.

Microsoft. Ciudades Digitales. Disponible en:

http://www.microsoft.com/spain/aapp/articulos/ciudades.mspx (Consultado el 29 de septiembre de 2010).

Motorola. Ranking Motora de Ciudades Digitales. Disponible en:

http://business.motorola.com/americalatina/rankingciudadesdigitales/index.html (Consultado el 29 de septiembre de 2010).

Muraro, H. (1987). Invasión cultural, economía y comunicación. Buenos Aires: Legasa.

Rabotnikof, N. (1997). El espacio público y la democracia moderna. México: Instituto Federal Electoral.

Schmucler, H. \& Terrero, P. (1991). Nuevas tecnologías y transformaciones del espacio urbano. Buenos Aires (1970-1990)". Ponencia presentada en el Coloquio Internacional 
Grandes Metrópolis de África y de América Latina. Toulouse: ILET 1991.

Schmucler, H. (1997). Memorias de la comunicación. Buenos Aires: Biblos.

Sennett, R. ([1974] 2002). El declive del hombre público. Barcelona: Península.

Varela, A. (2003). La ciudad. Un posible mapeo de la mirada. En Saintout, F. Abrir la Comunicación. Tradición y Movimiento en el campo académico. La Plata. EPC. Pp. 147-155.

Verón, E. (1987). La palabra adversativa. Observaciones sobre la enunciación política. En AA.VV. El discurso político. Lenguajes y acontecimientos Buenos Aires: Hachette. Pp. 11-25.

Verón, E. (1997). La semiosis social. Buenos Aires: Gedisa.

Ordenanza No8.052 Municipalidad de Rosario. Consultado el 20 de Septiembre de 2010), Disponible en:

http:/ / www.rosario.gov.ar/normativa/verArchivo?tipo=pdf\&id=43616

Proyecto Rosario 2010 Ciudad Digital. Consultado el 20 de Septiembre de 2010, Disponible en:

http://www.rosario.gov.ar/sitio/gobierno/gestion/ciudad_digital1.jsp.

Proyecto Autopista de la Información. Consultado el 20 de Septiembre de 2010, Disponible en:

http://www.aui.edu.ar/AUIasp/Index.asp .

Proyecto San Luis Digital 2010. Consultado el 20 de septiembre de 2010, Disponible en: http://www.sanluisdigital.edu.ar/SLDasp/paginas/pagina.asp?PaginaSLDID=14.

\section{Ana Gabriela Yeremian}

Licenciada en Comunicación Social y docente de la Universidad Blas Pascal. Especialista en Investigación de la Comunicación en el Centro de Estudios Avanzados (CEA) y doctoranda en el Doctorado en Estudios Sociales de América Latina (CEA), becaria doctoral del Consejo Nacional de investigaciones científicas y técnicas (CONICET). Es miembro del Programa de Investigación "Estudios Interdiscipliarios sobre juventud, comunicación y prácticas educativas emergentes" (CEA-UNC). Ha publicado artículos y ponencias presentadas en congresos nacionales e internacionales sobre los temas: "espacio público- medios y tecnologías de la información" y "TICeducación escolar- política educativa”. 


\section{Georgina Lía Remondino}

Licenciada en Comunicación Social y profesora asistente en la Escuela de Ciencias de la Información de la Universidad Nacional de Córdoba (UNC). Especialista en Investigación de la Comunicación en el Centro de Estudios Avanzados (CEA) y doctoranda en el Doctorado en Estudios Sociales de América Latina (CEA), becaria doctoral del Consejo Nacional de investigaciones científicas y técnicas (CONICET). Es miembro e instructora del Observatorio de Jóvenes, medios y TICs de la Facultad de Psicología de la UNC. Es miembro investigadora del Programa “Transformaciones culturales contemporáneas" radicado en el (CEA-UNC). Es autora de capítulos de libros, artículos en revistas y ponencias presentadas en congresos nacionales e internacionales en la temática "Jóvenes-Comunicación-TICs". 